\title{
GESTIÓN DE LOS RECURSOS DOCUMENTALES EN ARTIUM
}

\section{Elena Roseras-Carcedo}

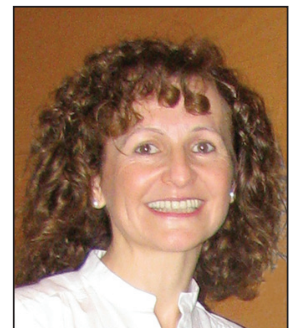

Elena Roseras-Carcedo es responsable de la Biblioteca y Centro de Documentación de Artium, Centro-Museo Vasco de Arte Contemporáneo. Licenciada en geografía e historia por la Universidad del País Vasco, licenciada en documentación y máster en sociedad de la información y del conocimiento por la Universitat Oberta de Catalunya (UOC). Es autora de varios artículos en publicaciones especializadas y ha participado como ponente en diversos congresos. http://orcid.org/0000-0001-6298-9828

Biblioteca y Centro de Documentación de Artium Centro Museo Vasco de Arte Contemporáneo Francia, 24. 01002 Vitoria-Gasteiz, España eroseras@artium.org

\section{Resumen}

Conscientes de la necesidad de una gestión óptima de los recursos documentales que ayude en la consecución de los objetivos que tiene marcados el Museo, se expone el trabajo que se está llevando a cabo desde la Biblioteca y Centro de Documentación de Artium. Se ofrece una visión general sobre la gestión del patrimonio bibliográfico, la creación de contenidos digitales a través de DokuArt y el desarrollo del archivo sonoro y de actividad del Museo.

\section{Palabras clave}

Gestión de contenidos, Archivo documental, Código abierto, Patrimonio bibliográfico, Drupal, Web social, Museos, Artium.

\section{Title: Documentary resource management in Artium}

\section{Abstract}

Aware of the need for the optimal management of documentary resources to help us fulfil the goals set by the museum, we are presenting the work being undertaken in this direction by the Artium Library and Documentation Centre. An overview is provided on bibliographic heritage management, digital content creation through DokuArt and the creation of sound archives and museum activity.

\section{Keywords}

Content management, Document file, Open source, Drupal, Social web, Bibliographic heritage, Museum, Artium.

Roseras-Carcedo, Elena (2013). "Gestión de los recursos documentales en Artium". El profesional de la información, mayo-junio, v. 22, n. 3, pp. 239-243.

http://dx.doi.org/10.3145/epi.2013.may.07

\section{Introducción}

Desde el centro de documentación se pretende abordar la gestión de la información como el pilar básico sobre el que se sustenta el conocimiento. Para ello es necesario delimitar los recursos documentales disponibles, intentando optimizar su rendimiento.

Dentro de esta línea de actuación, se están catalogando los tipos documentales, digitalizando parte del fondo y generando contenidos digitales que ayuden a una mayor comprensión y difusión de la cultura contemporánea.

\section{Dossiers de entidad}

Los museos, galerías, ferias de arte y, en general, instituciones culturales, generan una gran cantidad de material de carácter muchas veces efímero, pero que proporciona información de primera mano acerca de sus colecciones y de sus actividades temporales: folletos de exposiciones, material didáctico, postales, invitaciones, etc.

Consciente de la importancia de este tipo de documentación, Artium suscribió un acuerdo en 2002 -año de su inauguración- con la Feria de Arte Contemporáneo Arco, de forma que la organización de la Feria cedía a Artium toda la documentación que las galerías presentaban cada año. EI Museo, por su parte, se comprometía a gestionar dicha documentación para su conservación, tratamiento y difusión. Este acuerdo se mantuvo durante varios años y actualmente se sigue recogiendo la información que envían galerías, museos e instituciones culturales. La biblioteca recibe una documentación muy variada que requiere un tratamiento 
técnico diferenciado en función de su tipo: catálogos que las entidades editan con motivo de las exposiciones que organizan, folletos, postales, material didáctico, invitaciones, recortes de prensa... En ocasiones es muy heterogénea, y no se publica o no se distribuye por los cauces editoriales tradicionales.

Para su organización, en primer lugar se separa el material librario del resto, y pasa al servicio de catalogación. El no librario se clasifica alfabéticamente teniendo en cuenta la entidad que lo produjo, y posteriormente se realiza el tratamiento individual y exhaustivo de cada conjunto documental.

El dossier de entidad constituye una unidad documental indivisible, por lo que no se separa la información contenida en cada uno. Antes de abordar su descripción exhaustiva se organiza, de forma que los fondos tengan una estructura consistente.

En primer lugar se incluyen los documentos relativos a la entidad en general como planos, memorias de actividades, recortes de prensa..., ordenados siempre cronológicamente.

Seguidamente, separados del bloque anterior, se incluyen los documentos de las exposiciones celebradas por la entidad. Aquí tienen cabida las invitaciones y los recortes de prensa, siempre clasificados por años.

Por último, la documentación sobre artistas que la entidad representa: currícula vitae, fotografías de su obra o documentación de exposiciones llevadas a cabo en otras galerías, principalmente. Esta documentación se mantiene con el resto de la producida por la entidad y se ordena alfabéticamente por el apellido del artista.

Para gestionar este volumen ingente de documentación, se decidió integrarlo en el catálogo de la biblioteca, para mantener la coherencia de los fondos y, sobre todo, para facilitar al máximo la recuperación exhaustiva de la información.

El número de dossiers de artista y de entidad documentados asciende a 7.512 (abril 2013).

\section{Archivo documental de actividades}

Ante la necesidad de recoger toda la información sobre la actividad generada en el Museo desde que comenzó su andadura, se decidió crear una base de datos que permitiese acometer esta tarea de una manera estructurada y con vistas a poder sacar el máximo rendimiento al proyecto.

En primer lugar, era necesario saber el tipo de información que se iba a recoger y dónde se podía localizar. Se estableció una tipología de actividades: educativas, cine, conciertos, conferencias, cursos y talleres, escena contemporánea, especiales, exposiciones, participación en cursos, congresos y jornadas, programas para familias, programas para jóvenes, publicaciones, y visitas guiadas.

Se comenzó a establecer la estructura de la base de datos con los campos necesarios para la descripción de cada actividad: tipo de actividad, subtipo de actividad, serie, proceso asociado, exposición asociada, título, fecha-inicio, fecha-fin, descripción, director/comisario/autor, obra de la colección, año, país, diseñador, imprenta, número de páginas, espacio

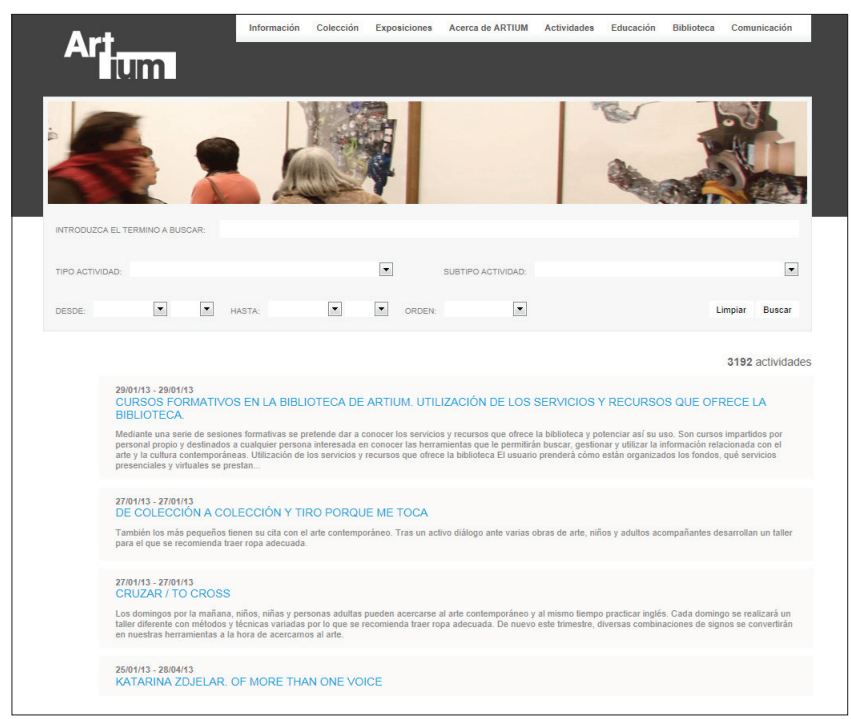

Figura 1. Archivo documental de actividades

expositivo, actividades relacionadas con la colección, idioma en que se desarrolla la actividad, hipervínculo, artículos de prensa, vídeo, fotografías, cartel, folleto, aforo y observaciones.

A través de estos campos se ha intentado aglutinar toda la actividad del Museo incluyendo la conexión con los artículos de prensa de cada actividad previamente catalogados, digitalizados y accesibles desde el catálogo bibliográfico. Así mismo se ofrece la conexión con las fotos previamente subidas a Flickr, con los vídeos subidos a YouTube y con los carteles y folletos de la actividad que están catalogados, digitalizados y accesibles a través del catálogo bibliográfico.

\section{DoKuArt es un archivo documental con dossiers sobre las exposiciones, artistas y escritores, monografías sobre películas y sus directores, archivo sonoro y pro- gramas de mano de cine}

Una vez recopiladas y documentadas las 3.258 actividades de los 10 primeros años (2002-12) se pensó que, además de ser una buena herramienta de gestión interna, podía ser interesante hacer llegar toda esta información a los usuarios a través de la web.

http://www.trepeta.es

\section{DokuArt}

Se trata de un archivo documental en el que pueden consultarse los dossiers elaborados sobre las exposiciones bibliográficas, artistas y escritores, monografías sobre películas y sus directores, el archivo sonoro y una colección de programas de mano de cine, procedentes de los programas y actividades organizados en el centro.

En un primer momento se elaboraron dossiers documentales que se ponían a disposición de los usuarios mediante páginas html estáticas creadas individualmente. Al ver la 
necesidad de gestionar de manera integral toda la información que se estaba generando, en 2005 se consideró la posibilidad de trabajar con un sistema de gestión de contenidos. Tras analizar las posibilidades, se optó por un gestor de contenidos de código abierto y que se pudiese desarrollar desde la propia biblioteca (Engard, 2010; Farkas, 2007; Tramullas; Garrido-Picazo; Sánchez-Casabón, 2011), y Drupal pareció una plataforma adecuada.

Su sencilla interfaz y sus características permiten gestionar los contenidos de forma eficiente. Es un producto estable, escalable y sobre todo de muy fácil utilización. Se pueden incluir textos, imágenes, blogs, vídeos, eventos, calendarios, encuestas, trabajo colaborativo, etc. Su arquitectura extensible permite añadir rápidamente nuevo contenido, nuevos temas y mejoras en la funcionalidad (Esteban-Navarro, 2006).

Durante estos años se han creado las diferentes secciones y elaborando los dossiers que documentan la actividad del centro. La tabla 1 muestra el cronograma seguido.

A mediados de 2012 se decidió actualizar los módulos de Drupal, crear nuevas secciones, modificar la estructura, incorporar nuevas funciones en las distintas secciones y cambiar completamente el diseño del programa adaptándolo a la estética de la web del Museo (Vacas-Guerrero; BonillaSánchez, 2011).

Se ha relacionado la información de DokuArt con el catálogo bibliográfico, de modo que dentro del apartado Recursos en nuestro centro de documentación la signatura de cada referencia bibliográfica enlaza con el registro del documento referenciado. Igualmente, dentro del catálogo, cuando hay documentación sobre algún artista, película u otra información que está disponible en DokuArt se enlaza el registro con el dossier correspondiente.

En DokuArt se trabaja con las redes sociales para integrar toda la información asociada al dossier que se está documentando. Cuando el Museo organiza una exposición, ciclo de cine, conferencia, concierto, etc., se usan las redes so-
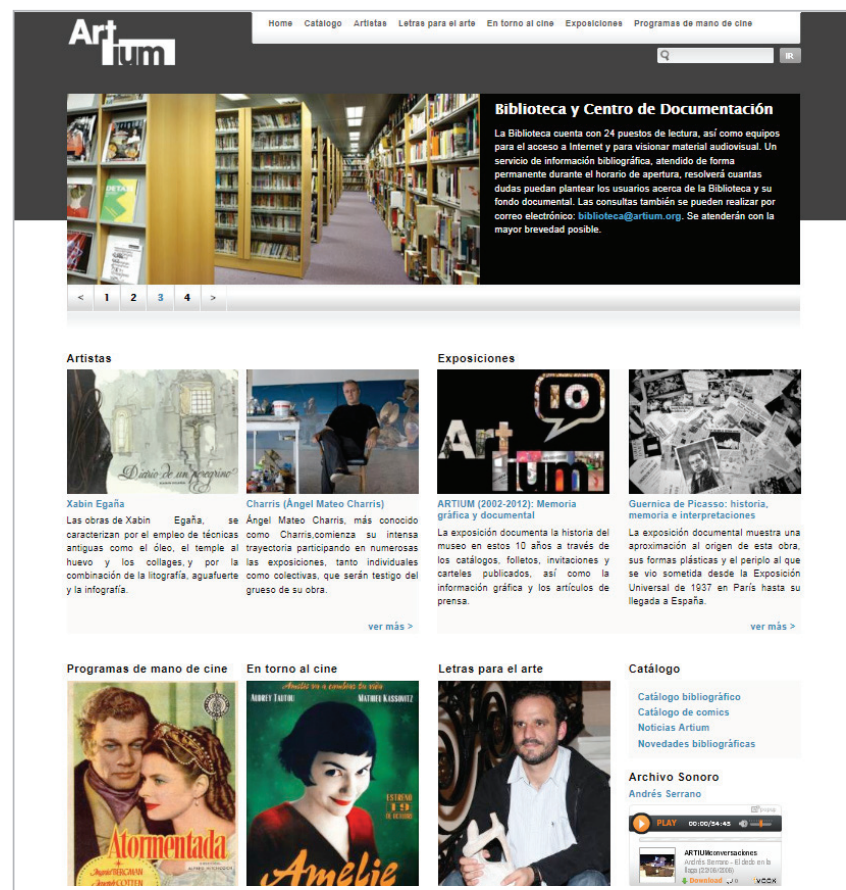

Figura 2. DoKuart. Página principal

ciales para difundir la actividad e involucrar a los usuarios en el programa. Se crean álbumes de fotos que se suben a Flickr, se comparten los vídeos en YouTube y se anuncian en Twitter y Facebook. Así mismo, para difundir las colecciones bibliográficas y la información de DokuArt se ha creado un tablón de contenidos en Pinterest (Gómez-Pereda; MerloVega, 2010; González-Vilches, 2012; González-FernándezVillavicencio, 2013).

Las secciones establecidas en el gestor de contenidos DokuArt son las siguientes:

http://catalogo.artium.org

\subsection{Artistas}

A través de un índice alfabético se ofrece información sobre los artistas que forman parte de la colección museográfica

\begin{tabular}{|c|c|c|c|c|c|c|c|c|c|}
\hline Tareas & 2005 & 2006 & 2007 & 2008 & 2009 & 2010 & 2011 & 2012 & 2013 \\
\hline Auditoría de la información y análisis de soluciones tecnológicas & & & & & & & & & \\
\hline Elección de Drupal y análisis y planificación del gestor de contenidos & & & & & & & & & \\
\hline Diseño y estructura del gestor de contenidos & & & & & & & & & \\
\hline Definición de perfiles de usuarios y niveles de seguridad & & & & & & & & & \\
\hline Pruebas y valoración del gestor de contenidos & & & & & & & & & \\
\hline Migración de dossiers ya elaborados a Drupal & & & & & & & & & \\
\hline Elaboración de nuevos dossiers documentales & & & & & & & & & \\
\hline $\begin{array}{l}\text { Planificación de las copias de seguridad y de la preservación de } \\
\text { los contenidos digitales }\end{array}$ & & & & & & & & & \\
\hline Evaluación del gestor de contenidos & & & & & & & & & \\
\hline $\begin{array}{l}\text { Cambio en la estructura, el diseño e inclusión de nuevas funcio- } \\
\text { nes en Drupal }\end{array}$ & & & & & & & & & \\
\hline
\end{tabular}

Tabla 1. Cronograma 


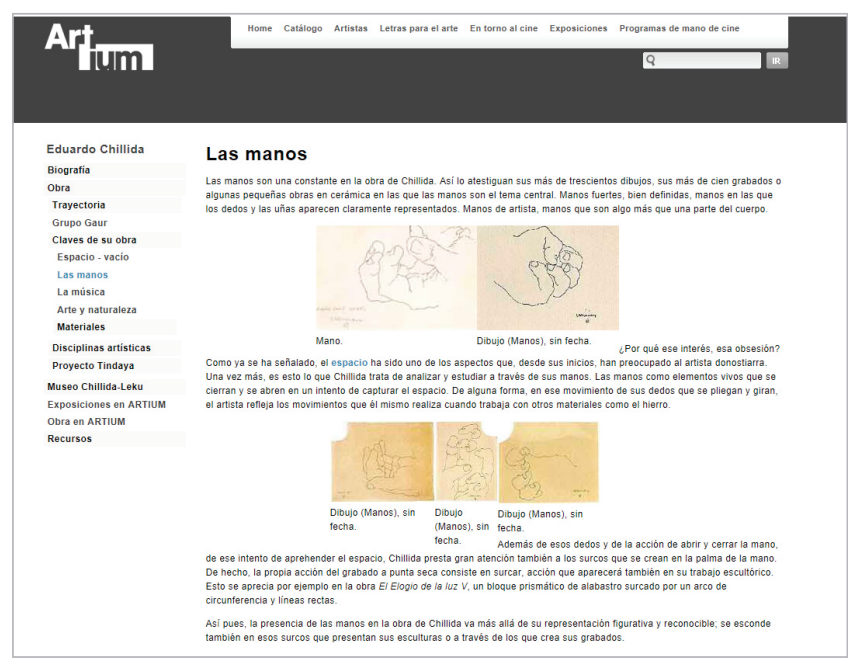

Figura 3. DoKuart. Artistas. Eduardo Chillida

o que han participado en alguna exposición de Artium. Dependiendo de la información que se tenga sobre el artista se habilitan subapartados pero, en general, se ofrece información sobre su biografía, obra, exposiciones y obra en Artium, y recursos. Este último apartado engloba los recursos online, bibliografía y materiales del centro de documentación.

\subsection{Exposiciones}

Las exposiciones se documentan para dar a conocer los fondos documentales del Museo y ofrecer las herramientas necesarias para que los usuarios puedan investigar sobre las mismas y sus temas.

\subsection{En torno al cine}

En el Museo se organizan ciclos de cine, tertulias de película, etc. En este apartado y, a través de un alfabético por directores, actores y películas, se ofrece información sobre algunas de las películas programadas, como la ficha técnica, sinopsis de la película, análisis crítico, premios recibidos, e información sobre el director y actores principales.

\subsection{Letras para el arte}

Actividad que pretende unir el arte y la literatura a través de la mirada de escritores, a los que se les solicita elegir una

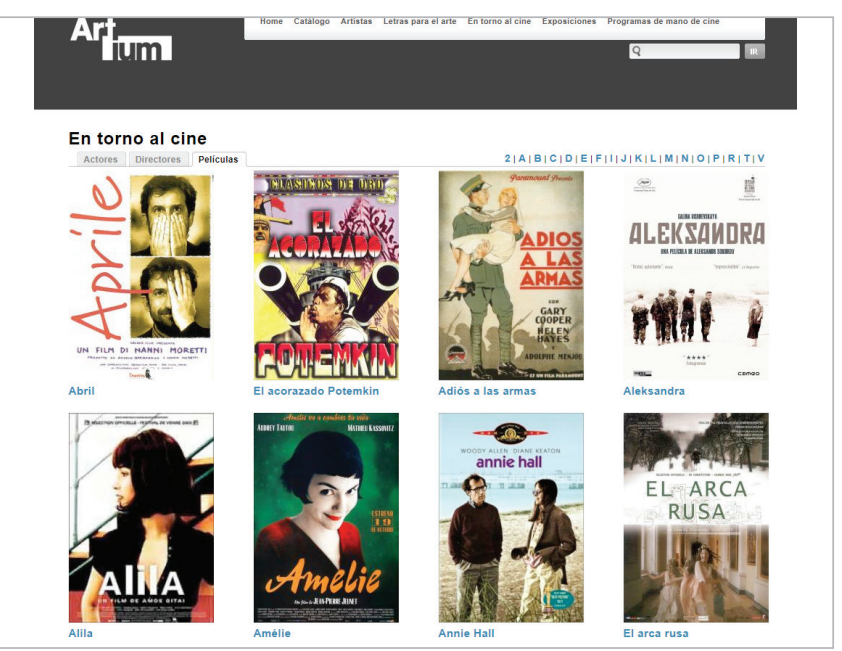

Figura 5. DoKuart. En torno al cine

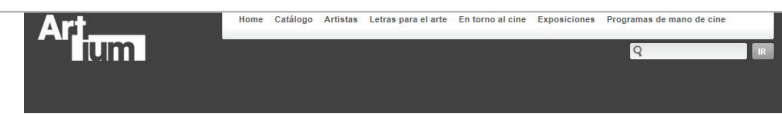

Exposiciones
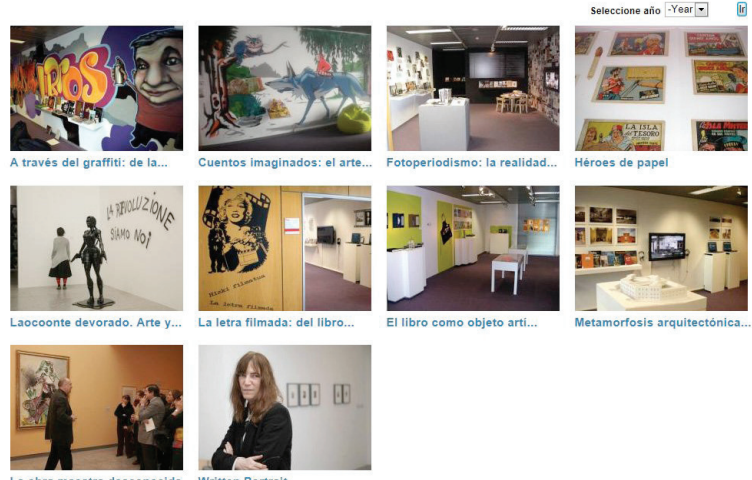

Figura 4. DoKuart. Exposiciones

obra de la colección del Museo y a ofrecer su visión personal sobre ella. Desde 2005, año de puesta en marcha de este programa, lo han realizado 45 escritores. Se consideró necesario tener documentada esta actividad y ponerla a disposición de todos los usuarios. Por ello, mediante un alfabético por escritores y artistas se ofrece información sobre ambos y el enlace a la conferencia del escritor, que previamente se ha subido a YouTube.

\subsection{Programas de mano de cine}

El Centro cuenta con una colección de un millar de programas de mano de cine de los años 30 al 60 que se ha digitalizado, documentado y puesto a disposición de todos los usuarios. En un primer momento se documentaron con Portfolio pero, conscientes de la necesidad de integrarlos con toda la información que se está generando, se ha creado en DokuArt esta nueva sección donde se ofrece la ficha de cada programa, el acceso a la imagen digitalizada y el tráiler de la película.

\section{Archivo sonoro}

En el Museo se organizan conversaciones con artistas, conferencias y entrevistas a personajes colaboradores. Se trata de un testimonio sonoro fundamental para la reconstruc-

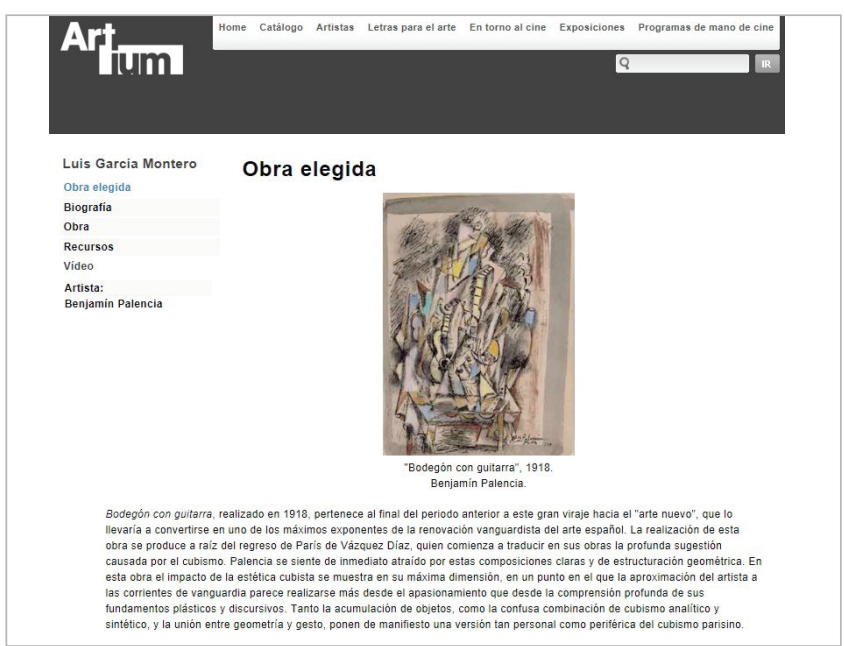

Figura 6. DoKuart. Letras para el arte. Luis García Montero 

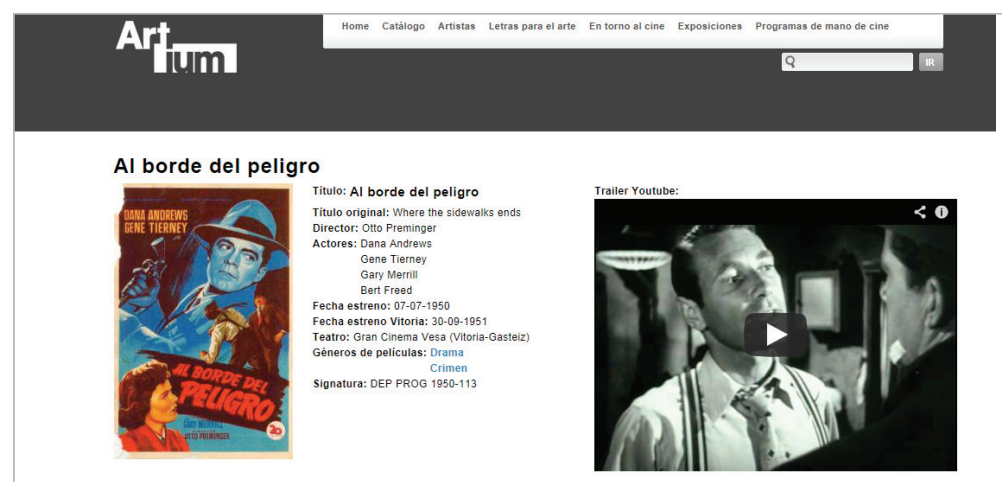

Figura 7. DoKuart. Programas de mano de cine

ción de la historia del Museo y que es necesario preservar, catalogar y, al mismo tiempo poner a disposición de todas las personas interesadas.

Por ello, en primer lugar se ha realizado un estudio de la colección y se ha establecido un flujo de trabajo que permita acometer el proyecto en base a unos criterios previamente establecidos. Se ha considerado el estado de conservación de los originales, la relevancia del contenido, la pertenencia de los artistas a la colección museográfica, y la conexión con los programas que actualmente se desarrollan en el Museo.

Seguidamente, se procede a la gestión de los derechos de autor y a la digitalización, edición y catalogación del fondo sonoro. Además de ofrecer la información a través del catálogo de la biblioteca, se ha creado un apartado en DokuArt que permite ofrecer este fondo de forma más directa.

Se pretende facilitar y fomentar el acceso al patrimonio cultural

\section{Catálogo}

Se ofrece el acceso al catálogo bibliográfico desarrollado con VuFind, un software de código abierto. Se han creado apartados para poder facilitar algunas consultas como catálogo de comics, noticias Artium y novedades bibliográficas.

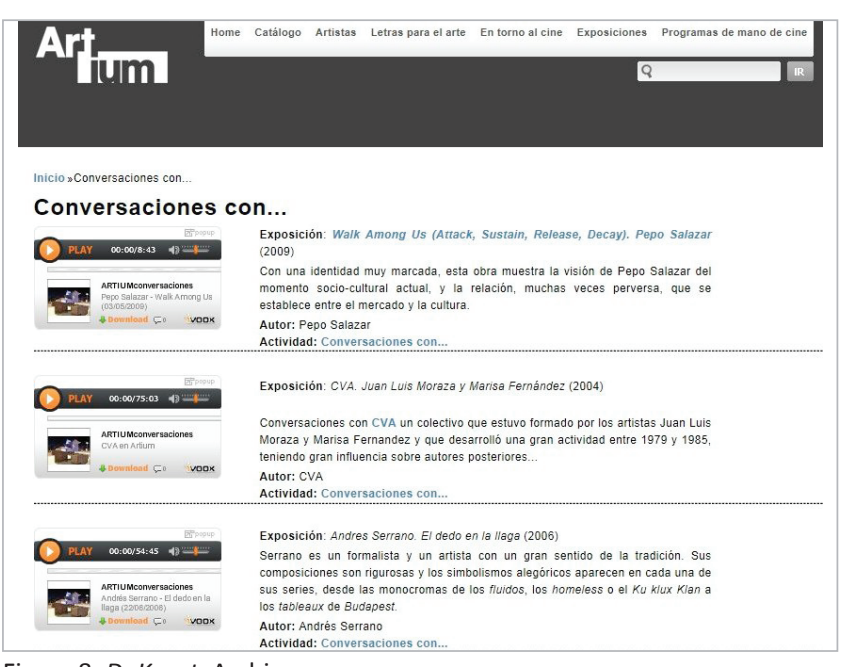

Figura 8. DoKuart. Archivo sonoro
Hay 90.717 registros bibliográficos. http://biblioteca.artium.org

Número de dossiers incluidos hasta este momento en DokuArt:

-Artistas: 77

-Escritores: 37

-Películas: 48

-Directores de cine: 35

-Actores / actrices: 105

-Exposiciones: 15

-Programas de mano de cine: 31

-Archivo sonoro: 23

Total: 371

\section{Conclusión}

Mediante la gestión del patrimonio documental se articulan los procesos de construcción de contenidos y las posibilidades comunicativas, optimizando el uso de los productos culturales. Así se facilita y fomenta el acceso al mismo y se crean sinergias entre los elementos del Museo.

\section{Bibliografía}

Engard, Nicole C. (2010). Practical open source software for libraries. Oxford: Chandos Publishing. ISBN: 1843345854

Esteban-Navarro, Miguel-Ángel (2006). “Planificación, diseño y desarrollo de servicios de información digital". En: Tramullas, Jesús; Garrido-Picazo, Piedad (coords.). Software libre para servicios de información digital. Madrid: Pearson Prentice Hall, pp. 23-44. ISBN: 9788483222997 http://eprints.rclis.org/7495

Farkas, Meredith G. (2007). Social software in libraries: building collaboration, communication, and community online. Medford, NJ: Information Today. ISBN: 9781573872751

Gómez-Pereda, Noemí; Merlo-Vega, José-Antonio (coords.) (2010). "Experiencias bibliotecarias con las tecnologías sociales". Educación y biblioteca, mayo-junio, n. 177, pp. 59125.

http://www.baratz.es/portals/0/noticias/Dossier\%20 Educación\%20y\%20Biblioteca.pdf

González-Vilches, Soledad (2012). "Museos españoles y redes sociales". Telos, Revista de la Fundación Telefónica, n. 91.

http://goo.gl/Qmquh

González-Fernández-Villavicencio, Nieves (2013). “Pinterest y las bibliotecas". Anuario ThinkEPI, v. 7, pp. 64-71. http://thinkepi.net

Tramullas, Jesús; Garrido-Picazo, Piedad; Sánchez-Casabón, Ana-Isabel (2011). “Groupware y software social: una propuesta de marco de evaluación analítico sobre herramientas de software libre". El profesional de la información, julio-agosto, v. 4, n. 20, pp. 465-473. http://dx.doi.org/10.3145/epi.2011.jul.16

Vacas-Guerro, Trinidad; Bonilla-Sánchez, Eusebio (coords.) (2011). Museos y comunicación. Un tiempo de cambio. Madrid: Universidad Rey Juan Carlos. ISBN: 9788497722995 